\title{
A perfect-fluid spacetime for a slightly deformed mass
}

\author{
M. Abishev, K. Boshkayev, H. Quevedo and S. Toktarbay* \\ Physical-Technical Faculty, Al-Farabi Kazakh National University, \\ Al Farabi av. 71, 050040 Almaty, Kazakhstan \\ Instituto de Ciencias Nucleares, Universidad Nacional Autónoma de México, \\ AP 70543, México, DF 04510, Mexico \\ *E-mail: saken.yan@yandex.com
}

\begin{abstract}
We present approximate exterior and interior solutions of Einstein's equations which describe the gravitational field of a static deformed mass distribution. The deformation of the source is taken into account up to the first order in the quadrupole.
\end{abstract}

Keywords: Quadrupole, compact objects, perfect fluid

To describe the gravitational field of a static axially symmetric mass distribution in general relativity, it is necessary to consider the multipole moments of the source. From a physical point of view, one expects that the quadrupole is the largest contributor and higher multipoles can be neglected in a first approximation. In this case, to describe the exterior field one can use, for instance, the exact quadrupole metric $(q-\text { metric })^{1,2}$.

$$
\begin{aligned}
d s^{2} & =A^{1+q} d t^{2}-A^{-q} \\
& \times\left[\left(1+\frac{m^{2} \sin ^{2} \theta}{r^{2} A}\right)^{-q(2+q)}\left(\frac{d r^{2}}{A}+r^{2} d \theta^{2}\right)+r^{2} \sin ^{2} \theta d \varphi^{2}\right],
\end{aligned}
$$

with $A=1-2 m / r$, which has been shown to be the simplest generalization of the Schwarzschild metric containing a quadrupole parameter $q$. Considering the quadrupole up to the first order only, we obtain

$$
\begin{aligned}
d s^{2} & =A(1+q \ln A) d t^{2}-r^{2} \sin ^{2} \theta(1-q \ln A) d \varphi^{2} \\
- & {\left[1+q \ln A-2 q \ln \left(A+\frac{m^{2}}{r^{2}} \sin ^{2} \theta\right)\right]\left(\frac{d r^{2}}{A}+r^{2} d \theta^{2}\right) . }
\end{aligned}
$$

This is an approximate solution of Einstein's vacuum equations up to the first order in $q$. The total mass of the spacetime turns out to be $M_{0}=$ $m(1+q)$ and the quadrupole moment is $M_{2}=-(2 / 3) q m^{3}$. 
The interior solution can be generated by using the method proposed recently in Ref. 3. We obtain

$$
\begin{aligned}
& d s^{2}=e^{2 \psi_{0}}\left(1+2 \tilde{q} \psi_{0}\right) d t^{2}-e^{-2 \psi_{0}}\left(1-2 \tilde{q} \psi_{0}\right) \\
& \times\left[e^{2 \gamma_{0}}\left(1+4 \tilde{q} \gamma_{0}+\tilde{q} \gamma_{1}\right)\left(\frac{d r^{2}}{r^{2} f^{2}(r)}+r^{2}\left(\sin ^{2} \theta-\tilde{q} \sin \theta \cos \theta\right) d \varphi^{2}\right)\right] \\
& e^{2 \psi_{0}}=\frac{3}{2} f(R)-\frac{1}{2} f(r), f(r)=\sqrt{1-\frac{2 \tilde{m} r^{2}}{R^{3}}}, e^{\gamma_{0}}=r e^{2 \psi_{0}} \\
& \gamma_{1}=-2 \int \frac{1+4 \pi \sin ^{2} \theta r^{2} p_{0}}{r f^{2}(r)\left(1+r \psi_{0, r}\right) \sin ^{2} \theta+\frac{r}{1+r \psi_{0, r}} \cos ^{2} \theta} d r+\kappa \\
& \psi_{0, r}=\frac{2 \tilde{m} r}{R^{3} f(r)[3 f(R)+f(r)]}
\end{aligned}
$$

where $\tilde{m}, \tilde{q}, R$ and $\kappa$ are real constants. This is an interior solution up to the first order in $\tilde{q}$ for a perfect fluid with density and pressure

$$
\begin{aligned}
& \rho=\rho_{0}\left[1+\tilde{q}\left(1+\psi_{0}-4 \gamma_{0}-\gamma_{1}\right)\right], \rho_{0}=\text { const. } \\
& p=p_{0}\left[1+\tilde{q}\left(1+\psi_{0}-4 \gamma_{0}-\gamma_{1}\right)\right], p_{0}=\rho_{0} \frac{f(r)-f(R)}{3 f(R)-f(r)},
\end{aligned}
$$

respectively. In the limiting case $\tilde{q} \rightarrow 0$, the metric (3) represents a perfect fluid with constant density $\rho_{0}$ and pressure $p_{0}$ as given in Eq.(8). If $\tilde{m}=m$, this particular solution can be matched with the exterior Schwarzschild metric along a sphere of radius $R$.

In the general case $\tilde{q} \neq 0$, a more detailed analysis must be carried out in order to match the above approximate interior solution with the approximate exterior $q$-metric given in Eq.(2). First, the matching surface must be established. Then, the matching conditions must be imposed for all metric components. This would imply a relationship between the exterior parameters $m$ and $q$ and the interior parameters $\tilde{m}, \tilde{q}, \rho_{0}$ and $\kappa$. This result will be presented elsewhere.

We acknowledge the support through a Grant of the Target Program of the MES of the RK, Grant No. 1597/GF3 IPC-30, DGAPA-UNAM, Grant No. 113514, and Conacyt, Grant No. 166391.

\section{References}

1. H. Quevedo, Int. J. Mod. Phys. D 20, 1779 (2011).

2. D. M. Zipoy, J. Math. Phys. 7 (1966) 1137; B. Voorhees, Phys. Rev. D 2 (1970) 2119.

3. H. Quevedo and S. Toktarbay, J. Math. Phys. 56, 052502 (2015). 